\title{
Drug delivery: Experiments, mathematical modelling and machine learning Boso et al.
}

(https://www.ncbi.nlm.nih.gov/pmc/articles/PMC8317640/pdf/nihms-1630189.pdf)

In this article, scientists from two Italian universities developed a model for drug delivery in order to determinate the efficacity of anticancer therapy by using Machine Learning and Artificial Neural Networks. On a more experimental level, one of the main differences of this model compared to those already existing is the generation of 3D structure models instead of 2D ones, which represent more accurately the nature of tumors.

Mathematically, they took as a basic unit the mass and volume of each phase that composes a tumor: extracelullar matrix (as an only solid phase), healthy cells, interstitial fluid and tumor cells, considering that the volume occupied by the extracelular matrix is the complementary of porosity. Firstly, in order to build the model, these researchers designed several balance equations, in each of which every addend meant the possible changes between phases considering cell growth and lysis phenomena in a tumor. It was also assumed that healthy cells mass did not change, as it was considered to be in homeostasis.

$$
\begin{aligned}
& \varepsilon^{s}+\varepsilon^{h}+\varepsilon^{t}+\varepsilon^{l}=1 \quad \stackrel{l \rightarrow s}{M} \\
& \frac{\partial \varepsilon}{\partial\left(\varepsilon^{t} \rho^{t}\right)}=\nabla \cdot \mathbf{v}^{\bar{s}}+\frac{(1-\varepsilon)}{l \rightarrow t} \frac{\partial \rho^{s}}{t \rightarrow l}-\nabla \cdot\left(\varepsilon \mathbf{v}^{\bar{s}}\right)-\frac{\underset{E C M}{M}}{\rho^{s}} \\
& \frac{\partial\left(\varepsilon^{t} \rho^{t}\right)}{\partial t}+\nabla \cdot\left(\varepsilon^{t} \rho^{t} \mathbf{v}^{t}\right)=\underset{\text { growth }}{M}-\underset{M}{\text { lysis }}
\end{aligned}
$$

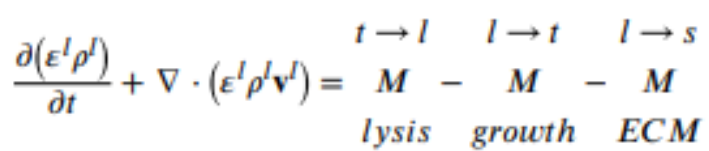

On the contrary, tumor cells were unfolded in two categories: living tumor cells and dead tumor cells, adding a new substracting factor: the death rate of tumor cells, obtaining two new equations: one expresses the mass balance for living cells (can grow or die) and the second one gives the same parameter but for necrotic cells (can appear or suffer from lysis). Adding diffusivity and considering oxigen and the drug as the nutrients (or $\mathrm{Nt}$ ), they obtained these equations.

$$
\begin{gathered}
\frac{\partial\left[\varepsilon^{t} \rho^{t}\left(1-\omega^{N \bar{t}}\right)\right]}{\partial t}+\nabla \cdot\left[\varepsilon^{t} \rho^{t}\left(1-\omega^{N \bar{t}}\right) \mathbf{v}^{\bar{t}}\right]=\underset{\text { grouth }}{\stackrel{l}{M}}-\varepsilon^{t} r^{N t} \\
\frac{\partial\left(\varepsilon^{t} \rho^{t} \omega^{N \bar{t}}\right)}{\partial t}+\nabla \cdot\left(\varepsilon^{t} \rho^{t} \omega^{N \bar{t}} \mathbf{v}^{\bar{t}}\right)=\varepsilon^{t} r^{N t}-\underset{\text { lysis }}{\stackrel{l}{M}}
\end{gathered}
$$


Latter combinations offer a last equation, of which only the last term was used as a basis for machine learning, which expresses the death rate of the drug, with $\lambda$ being the rate of drug induced cell death, $f$ being the activation function, which can have values of 0 or 1 based on the state of inactivation or activation of the drug, and $\omega$ being the mass fraction of the drug or necrotic cells. The model resulted to be accurate when predicting cell tumor growth in the abscense of drug or under low concentrations of it, but disturbing phenomena like activation time made it imposible to use the same prediction for higher amounts of drug, as drug induced cell death was not accurately calculated.

$$
\begin{gathered}
\varepsilon^{t} r^{N^{t}}=-\left[\gamma_{\text {necrosis }}^{t}\left(\frac{\omega^{\overline{n l}}-\omega_{\text {crit }}^{\overline{n l}}}{\omega_{\text {env }}^{\bar{n}}-\omega_{\text {crit }}^{\overline{n l}}}\right]_{-}-\delta_{a}^{t} H\left(p^{t}-p_{\text {necr }}^{t}\right)\right]\left(1-\omega^{N \bar{t}}\right) \varepsilon S^{t} \\
-\lambda_{\text {drug }} \omega^{d r u g}\left(1-\omega^{N \bar{t}}\right) \varepsilon^{t} f \\
\left(\varepsilon^{t} r^{N t}\right)_{\text {kill }}=\lambda_{\text {drug }} \omega^{d n u g}\left(1-\omega^{N t}\right) \varepsilon^{t} f
\end{gathered}
$$

It is here where machine learning is helpful by using artificial Neural Networks in order to calculate $\lambda$ as a function of drug concentration ( $\omega$ drug), tumor radius and time. In this new method, it is assumed that drug uptake did not depend on its concentration, as it was proved experimentally. As it happens sometimes when using $M L$, raw experimental data were not enough, so these had to be enriched by computational calculation of "more points", giving a total of 178 training set patterns and 20 patterns for test set after many attempts until the prediction of drug death rate $\lambda$ was accurate enough. The best topology for the Neural Network was of three layers of 3, 2 and 2 nodes, respectively. In this terms, ANN was very accurate when testing and predicted $\mathrm{a} \lambda$ of $5 \cdot 10^{6}$.

This experiment proves once again the necessity of improving ML models, as they are not accurate enough with a low number of training samples, but it gives promising results for its applications.

1. Boso DP, Di Mascolo D, Santagiuliana R, Decuzzi P, Schrefler BA. Drug delivery: Experiments, mathematical modelling and machine learning. Comput Biol Med. agosto de 2020;123:103820. 